\title{
No More Shiny Apples
}

\author{
MATTHEW I. CAMPBELL \\ University of Texas at Austin, Austin, Texas, USA
}

One could look at the last 10 years of AI in design and manufacturing as a period of refinement. Like the field of $\mathrm{AI}$ as a whole, the last decade has failed to produce any grand new theories. Rather, the research accomplishments are based more on moving slowly from toy problems to real-world situations. I vaguely recall a quote in a popular AI text from the 1990s that suggested that if you needed more than two heuristics to solve a problem, it might as well be an infinite number of heuristics. Such a defeatist attitude was possible 25 years ago because the tree of achievements contained many low-hanging fruits. These tempting, shiny apples of achievement led many popular science journalists to erroneously extrapolate how all of life's tedium would be offloaded to our AI lackies, but inevitably this vision shrouded many of the challenges that researchers are slogging through today.

Two heuristics are not enough, and likely there is a large number needed to catch up with the reasoning of natural intelligence. The past decade has seen a huge expanse in scientific knowledge about how the brain works, and one thing that can be tenuously gleaned from that work is that the human mind is capable of storing a vast amount of heuristics. There is plenty to be done between 2 and infinity; and as we move away from toy problems to real life, we find that a significant amount of effort is required to augment our simple yet powerful theories into useful tools.

Consider path planning. Textbook examples like moving through a simple maze relay the fundamental methods at the heart of "commercial strength" path planning, for example, using online mapping tools (like maps.google.com) to plan a trip from an airport to a hotel. These tools are nearly perfect today as they consider the intricacies of traffic, lane closures, tolls, and so forth. A decade ago, one could hardly trust the suggested routes. At that time, it was generally accepted that these online mapping tools would forever lack the common sense that humans honed during real travel. Our minds could distill the near-infinite sensory experiences into substantial, yet intangible, common sense heuristics.

The defeatist view continues both with experts and users. When a technology like online mapping reaches maturity, no one notices or bothers to adjust their opinions about how irreparably simple $\mathrm{AI}$ is. What is exciting and groundbreaking in design and manufacturing today? Would the readers of this Journal even put AI before nanotechnology? The fruits of our labor are no longer shiny apples, but raisins.

However, a raisin is sophisticated, intricate, and multifaceted. This period of refinement is needed to reach the simulacrum of human reasoning that is achieved in online path planning. We need to look past the programming of a few obvious heuristics and define ways to net more heuristicsmore of the rules of thumb that are used in design, analysis, and manufacturing. This will allow us to create "commercial strength" tools to truly alleviate tedious aspects of design, analysis, and manufacturing. How can we do this? In the past decade, the greatest expanse of computational innovation has occurred with graphic user interfaces and social networking, neither of which sounds terribly compelling; but perhaps both could be leveraged to catch the substantial but intangible design and manufacturing heuristics. There is a hive-mind of engineers who are comfortable with these innovations and can be enlisted to build the large (read: $>2$ ) bank of heuristics that are used by natural intelligence to design and manufacture. 